\title{
DIVISION I - Fundamental Astronomy
}

URL: < astro.cas.cz/iaudiv1>

\section{President \\ Jan Vondrák}

Astronomical Institute

Academy of Sciences

Bocn II 1401

CZ-141 31 Praha 4

Czech Republic

Phone: +420 267103043

Fax: +420272 769023

Email: <vondrak@ig.cas.cz>

\section{Vice-President}

Dennis D. McCarthy

US Naval Observatory (USNO)

3450 Massachusetts Avenue NW

Washington, DC 20392-5420

USA

Phone: +1 2027621837

Fax: +1 2027621563

Email: <dmc@maia.usno.navy.mil>

\section{Organizing Committee}

Aleksander Brzezinski, P-C19 (Poland), Joseph A. Burns, P-C7 (USA), Pascale Defraigne, P-C31 (Belgium), Dafydd Wyn Evans, VP-C8 (UK) Toshio Fukushima, P-C4, PP (Japan), George H. Kaplan, VP-C4 (USA), Sergei A. Klioner, P-C52 (Germany), Zoran Knezevic, VP-C7 (Serbia) Irina I. Kumkova, P-C8 (Russia), Chopo Ma, VP-C19 (USA), Richard N. Manchester, VP-C31 (Australia), and Gérard Petit, VP-C52 (France)

\section{PARTICIPATING COMMISSIONS AND COMMISSION WORKING GROUPS}

\section{Div.I/Comm.4 - Ephemerides}

P: Toshio Fukushima, PP, (Japan), <Toshio.Fukushima@nao.ac.jp>

VP: George H. Kaplan, (USA), < gkaplan@usno.navy.mil>

OC: Jan Vondrák (Czech Rep.), Catherine Hohenkerk (UK), John A. Bangert (USA), Sean E. Urban (USA), Jean-Eudes Arlot (France), Martin Lara (Spain), and Elena V. Pitjeva (Russia)

URL: <http://iau-comm4.jpl.nasa.gov/>

\section{Div.I/Comm.7 - Celestial Mechanics and Dynamical Astronomy}

P: Joseph A. Burns (USA), <jab16@cornell.edu>

VP: Zoran Knežević (Serbia), <zoran@aob.bg.ac.yu>

S: David Vokrouhlicky (Czech Rep.), <vokrouhl@mbox.cesnet.cz>

OC: Evangelia Athanassoula (France), C. Beauge (Argentina), B. Erdi (Hungary), A. Maciejewski (Poland), R. Malhotra (USA), Andrea Milani, PP (Italia), A. Morbidelli (France), S.J. Peale (USA), and Ji-Lin Zhou (China)

URL: <http://copernico.dm.unipi.it/comm7>

\section{Div.I/Comm.8 - Astrometry}

P: Irina I. Kumkova (Russia), <kumkova@iperas.nw.ru>

VP: Dafydd Wyn Evans (UK), <dwe@ast.cam.ac.uk>

OC: Alexandre H. Andrei (Brazil), Alain Fresneau (France), Imants Platais (USA), Petre P. Popescu (Rumania), Ralf-Dieter Scholz (Germany), Mitsuru Soma (Japan), Norbert Zacharias (USA), and Zi Zhu (China)

URL: <http://www.ast.cam.ac.uk/iau_comm8/> 
Div.I/Comm.8/WG - Densification of the Optical Reference Frame

Chair: Norbert Zacharias (USA), <nz@usno.navy.mil>

URL: <http://ad.usno.navy.mil/dens_wg/dens.html>

Div.I/Comm.19 - Rotation of the Earth

P: Aleksander Brzezinski (Poland), <alek@cbk.waw.pl>

VP: Chopo Ma (USA), <cma@virgo.gsfc.nasa.gov>

OC: Patrick Charlot (France), Pascale Defraigne (Belgium), Véronique Dehant (Belgium), Jean O. Dickey (USA), ChengLi Huang (China), Jean Souchay (France), and Jan Vondrák (Chech Republic)

URL: <http://www.astro.oma.be/IAU/>

Div.I/Comm.31 - Time

P: Pascale Defraigne (Belgium), <Pascale.Defraigne@oma.be $>$

VP: Richard N. Manchester (Australia), <Dick.Manchester@csiro.au>

OC: Mizuhiko Hosokawa (Japan), Sigfrido Leschiutta (Italia), Demetrios Matsakis (USA),

Gérard Petit (France), and Zhai ZaoCheng (China)

URL: <http://www.astro.oma.be/IAU/COM31/>

Div.I/Comm.52 - Relativity in Fundamental Astronomy

P: Sergei A. Klioner (Germany), <Sergei.Klioner@tu-dresden.de>

VP: Gérard Petit (France), <gpetit@bipm.org>

OC: Viktor A. Brumberg (Russia), Nicole Capitaine (France), Agnès Fienga (France), Toshio Fukushima (Japan), Bernard R. Guinot (France), Cheng Huang (China), François Mignard (France), Kenneth P. Seidelmann (USA), Michael H. Soffel (Germany), and Patrick T. Wallace (UK)

URL: <http://astro.geo.tu-dresden.de/RIFA>

\section{DIVISION I WORKING GROUPS}

Div.I/WG - Second Realization of International Celestial Reference Frame

Chair: Chopo Ma (USA), <cma@gemini.gsfc.nasa.gov>

URL: <http://rorf.usno.navy.mil/ICRF2/>

Div.I/WG - Numerical Standards in Fundamental Astronomy

Chair: Brian J. Luzum (USA), <bjl@maia.usno.navy.mil>

URL: <http://maia.usno.navy.mil/NSFA.html>

Div.I/WG - Astrometry by Small Ground-Based Telescopes

Chair: William Thuillot (France) <thuillot@imcce.fr >

URL: <http://www.imcce.fr/hosted_sites/iau_wgnps/astrom.html>

\section{DIVISION I INTER-DIVISION WORKING GROUPS}

Div.I-III/WG - Cartographic Coordinates and Rotational Elements

Chair: Brent A. Archinal (USA), <barchinal@usgs.gov>

URL: <http://astrogeology.usgs.gov/Projects/WGCCRE/>

Div.I-III/WG - Natural Satellites

Chair: Jean-Eudes Arlot (France), <Jean-Eudes.Arlot@obspm.fr>

URL: <http://www.imcce.fr/host/iau_wgnps/iauwg.html> 
DIVISION II - Sun and Heliosphere

URL: <http://www.iac.es/proyecto/iau_divii/IAU-DivII/main/index.php>

President

Donald B. Melrose

School of Physics A28

University of Sydney

Sydney, NSW 2006

Australia

Phone: +61 293514234

Fax: +61293517726

Email: <melrose@physics.usyd.edu.au >
Vice-President

Valentin Martínez Pillet

Instituto de Astrofsica de Canarias

Observatorio del Teide

C/ Vía Láctea s/n

E-38200 La Laguna, Tenerife

Spain

Phone: +34 922605237

Fax: +34 922605210

Email: <vmp@.iac.es>

\section{Secretary}

Lidia van Driel-Gesztelyi, VP-C10 (France), <Lidia.vanDriel@obspm.fr>

\section{Organizing Committee}

David F. Webb, PP (USA), James A. Klimchuk, P-C10 (USA), Alexander Kosovichev, VP-C12 (USA), Jean-Louis Bougeret, P-C49 (France), and Rudolf von Steiger VP-C49 (Swiss)

\section{PARTICIPATING COMMISSIONS}

\section{Div.II/Comm.10 - Solar Activity}

P: James A. Klimchuk (USA), <james.klimchuk@nrl.navy.mil>

VP: Lidia van Driel-Gesztelyi (France), <Lidia.vanDriel@obspm.fr>

S: Karel J. Schrijver (USA), <schryver@lmsal.com>

OC: Donald B. Melrose, PP (Australia), Lyndsay Fletcher (UK), Nat Gopalswamy (USA), Richard A. Harrison (UK), Cristina H. Mandrini (Argentina), Hardi Peter (Germany), Saku Tsuneta (Japan), Bojan Vrsnak (Croatia), and Jingxiu Wang (China) URL: <http://www.mssl.ucl.ac.uk/iau_c10/index.html>

\section{Div.II/Comm.12 - Solar Radiation and Structure}

P: Valentin Martínez Pillet (Spain), <vmp@iac.es>

VP: Alexander Kosovichev (USA), <sasha@quake.stanford.edu>

S: John T. Mariska (USA), <mariska@nrl.navy.mil>

OC: Martin Asplund (Australia), Thomas J. Bogdan (USA), Gianna Cauzzi (Italy), Jørgen Christensen-Dalsgaard (Denmark), Lawrence E. Cram Australia), Weiqun Gan (China), Laurent Gizon (Germany), Petr Heinzel (Czech R.), Marta G. Rovira (Argentina), and P. Venkatakrishnan (India)

URL: <http://www.iac.es/proyecto/iau_divii/IAU-DivII/main/index.php>

\section{Div.II/Comm.49 - Interplanetary Plasma and Heliosphere}

P: Jean-Louis Bougeret (France), <Jean-Louis.Bougeret@obspm.fr>

VP: Rudolf von Steiger (Swiss), < rudolf.vonsteiger@issibern.ch>

OC: Subramanian Ananthakrishnan (India), Hilary V. Cane (Australia), Nat Gopalswamy (USA), Stephen W. Kahler (USA), Rosine Lallement (France), Blai Sanahuja (Spain), Kazunari Shibata (Japan), Marek Vandas (Czech R.), Frank Verheest (Belgium), and David F. Webb, PP (USA) 


\section{DIVISION II WORKING GROUPS}

\section{Div.II/WG - Solar Eclipses}

Chair: Jay M. Pasachoff (USA), <jay.m.pasachoff@williams.edu> URL: <http://www.williams.edu/Astronomy/IAU_eclipses/>

\section{Div.II/WG - Solar and Interplanetary Nomenclature}

Chair: Edward W. Cliver (USA), <Edward.Cliver@hanscom.af.mil> URL: <http://www2.bc.edu/ haganmp/Nomenclature.htm>

\section{Div.II/WG - International Solar Data Access}

Chair: Robert Bentley (UK), <rdb@mssl.ucl.ac.uk> URL: <http://www.mssl.ucl.ac.uk/grid/iau/DivII_WG_IntDataAccess.html>

\section{Div.II/WG - International Collaboration on Space Weather}

Chair: David F. Webb (USA), <David.Webb@hanscom.af.mil>

URL: <http://www2.bc.edu/ haganmp/MAINpage> 
DIVISION III - Planetary Systems Sciences

URL: <http://www.ss.astro.umd.edu/IAU/div3/>

\section{President}

Edward L.G. Bowell

Lowell Observatory

Box 1149

1400 West Mars Hill Road

Flagstaff, AZ 86001

USA

Phone: +1 9287743358

Fax: +1 9287746296

Email: <ebowell@lowell.edu>

\author{
Vice-President \\ Karen J. Meech \\ Institute for Astronomy \\ University of Hawai'i Honolulu \\ 2680 Woodlawn Drive \\ Honolulu, HI 96822 \\ USA \\ Phone: +1 8089566828 \\ Fax: +1 8089569580 \\ Email: <meech@ifa.hawaii.edu>
}

\section{Secretary}

Guy J. Consolmagno, (Vatican State City), <brother_guy@mac.com>

\section{Organizing Committee}

Alan P. Boss, P-C51 (USA), Régis Courtin, P-C16 (France), Julio A. Fernández, P-C20 (Uruguay), Bo A.S. Gustafson, PP-C21 (USA), Walter F. Huebner, P-C15 (USA), A.Chantal Levasseur-Regourd (France), Mikhail Ya. Marov (Russia), Michel Mayor, P-C53 (Switzerland), Rita M. Schulz (Germany), Pavel Spurný, P-C22 (Czech Rep.), Giovanni B. Valsecchi, PP-C20 (Italy), Jun-Ichi Watanabe (Japan), Iwan P. Williams, PP (UK), and Adolf N. Witt P-C21 (USA)

\section{PARTICIPATING COMMISSIONS AND COMMISSION WORKING GROUPS}

\section{Div.III/Comm.15 - Physical Study of Comets and Minor Planets}

P: Walter F. Huebner (USA), <whuebner@swri.edu>

VP: Alberto Cellino (Italy), <Cellino@to.astro.it>

S: Daniel C. Boice (USA), <DBoice@SwRI.edu>

OC: Dominique Bockelee-Morvan (France), Yuehua Ma (China), Harold J. Reitsema (USA), Rita M. Schulz (Netherlands), Petrus M.M. Jenniskens (USA), Dmitrij F. Lupishko (Ukraine), and Gonzalo Tancredi (Uruguay)

URL: <http://iau15.space.swri.edu>

\section{Div.III/Comm.15/WG - Physical Study of Comets}

Chair: Tetsuo Yamamoto (Japan), <TY@lowtem.hokudai.ac.jp>

URL: <http://atlas.sr.unh.edu/IAU_Comm15/>

\section{Div.III/Comm.15/WG - Physical Study of Minor Planets}

Chair: Ricardo Gil-Hutton (Argentina), <RGilHutton@casleo.gov.ar>

URL: <http://atlas.sr.unh.edu/IAU_Comm15/>

\section{Div.III/Comm.16 - Physical Study of Planets and Satellites}

P: Régis Courtin (France), <Regis.Courtin@obspm.fr>

VP: Melissa A. McGrath (USA), <melissa.a.mcgrath@nasa.gov>

S: Luisa M. Lara (Spain), <lara@iaa.es>

OC: Carlo Blanco (Italy), Guy J. Consolmagno (Vatican City State), Leonid V. Ksanfomality (Russia), David Morrison (USA), John R. Spencer (USA), and Viktor G. Tejfel (Kazakhstan)

URL: <http://www.iaa.es/IAUComm16> 
Div.III/Comm.20 - Positions and Motions of Minor Planets, Comets and Satellites

P: Julio A. Fernández (Uruguay), <julio@fisica.edu.uy>

VP: Makoto Yoshikawa (Japan), <makoto@pub.isas.ac.jp >

S: Steve Chesley (USA), <steve.chesley@jpl.nasa.gov>

OC: Giovanni B. Valsecchi, PP (Italy), Yulia A. Chernetenko (Russia), Alan C. Gilmore (New Zealand), Daniela Lazzaro (Brazil), Karri Muinonen (Finland), Petr Pravec (Czech Republic), Timothy B. Spahr (USA), David J. Tholen (USA), Jana Tychá (Czech Republic), and Jin Zhu (China)

URL: <http://www.astro.uu.se/IAU/c20/>

\section{Div.III/Comm.20/WG - Motions of Comets}

Chair: Julio Fernández (Uruguay, <julio@fisica.edu.uy>

URL: <http://www.astro.uu.se/IAU/c20/wgcomet.html>

\section{Div.III/Comm.20/WG - Distant Objects}

Chair: Brian Marsden (USA), <bmarsden@cfa.harvard.edu>

URL: <http://www.astro.uu.se/IAU/c20/>

\section{Div.III/Comm.21 - Light of the Night Sky}

P : Adolf N. Witt (USA), < awitt@dusty.astro.utoledo.edu>

VP: Jayant Murthy (India), <jmurthy@yahoo.com>

OC: W. Jack Baggaley (New Zealand), Eli Dwek (USA), Bo A.S. Gustafson (USA), A.Chantal Levasseur-Regourd (France), Ingrid Mann (Japan), Kalevi Mattila (Finland), and Junichi Watanabe (Japan)

URL: <http://www.astro.ufl.edu/gustaf/IAUCom21/IAU_Com_21.html>

\section{Div.III/Comm.22 - Meteors, Meteorites and Interplanetary Dust}

P: Pavel Spurn (Czech R.), <spurny@asu.cas.cz>

VP: Jun-ichi Watanabe (Japan), <jun.watanabe@nao.ac.jp>

S: Jiri Borovicka (Czech Rep.), <borovic@asu.cas.cz>

OC: William J. Baggaley (New Zealand), Peter G. Brown (Canada), Guy J. Consolmagno (USA), Petrus M.M. Jenniskens (USA), Asta K. Pellinen-Wannberg (Sweden), Vladimir Porubcan (Slovakia), Iwan P. Williams (UK), and Hajime Yano (Japan)

URL: <http://meteor.asu.cas.cz/IAU/>

\section{Div.III/Comm.22/WG - Professional-Amateur Cooperation in Meteors}

Chair: Galina O. Ryabova (Russia), <ryabova@niipmm.tsu.ru>

URL: <http://www.asu.cas.cz/english/>

\section{Div.III/Comm.22/TF - Task Force for Meteor Shower Nomenclature}

Chair: Petrus M.M. Jenniskens (USA), <pjenniskens@mail.arc.nasa.gov>

URL: <http://meteor.asu.cas.cz/IAU/nomenclature.html>

\section{Div.III/Comm.51 - Bioastronomy}

P: Alan P. Boss (USA), <boss@dtm.ciw.edu>

VP: William M. Irvine (USA), <irvine@fcrao1.astro.umass.edu>

OC: Cristiano Cosmovici (Italy), Pascale Ehrenfreund (Netherlands), Karen J. Meech, PP (USA), David W. Latham (USA), David Morrison (USA), and Stephane Udry (Switzerland)

URL: < http://www.dtm.ciw.edu/boss/c51index.html>

\section{Div.III/Comm.53 - Extrasolar Planets}

P : Michel Mayor (Switzerland), <michel.mayor@obs.unige.ch> 
VP: Alan P. Boss (USA), <boss@dtm.ciw.edu>

OC: Paul Butler (USA), William B. Hubbard (USA), Philip A. Ianna (USA), Martin Kuerster (Germany), Jack J. Lissauer (USA), Karen J. Meech (USA), François Mignard (France), Allan Penny (UK), Andreas Quirrenbach (Germany), Jill C. Tarter (USA), and Alfred Vidal-Madjar (France)

\section{DIVISION III WORKING GROUPS}

Div.III/Service - Minor Planet Center (MPC)

Director: Timothy B. Spahr (USA), <tspahr@cfa.harvard.edu>

URL: <http://cfa-www.harvard.edu/iau/mpc.html>

Div.III/Committtee - Minor Planet Center Advisory Committee

Michael F. A'Hearn (USA), Steven R. Chesley (USA), Hans Rickman (Sweden), and Giovanni B. Valsecchi (Italy)

Div.III/WG - Committee on Small Bodies Nomenclature (CSBN)

Chair: Jana Tichá (Czech Republic), <jticha@klet.cz>

S: Brian Marsden (USA), <bmarsden@cfa.harvard.edu>

URL: <http://www.ss.astro.umd.edu/IAU/csbn/>

Div.III/WG - Planetary System Nomenclature (WG-PSN)

Chair: Rita M. Schulz (Netherlands), <rschulz@rssd.esa.int>

URL: <http://planetarynames.wr.usgs.gov/append1.html>

\section{DIVISION III INTER-DIVISION WORKING GROUPS}

Div.III-I/WG - Cartographic Coordinates and Rotational Elements

Chair: Brett A. Archinal (USA), <barchinal@usgs.gov>

URL: <http://astrogeology.usgs.gov/Projects/WGCCRE/\#wgm>

Div.III-I/WG - Natural Satellites (formerly Div.III- C20 WG)

Chair: Jean-Eudes Arlot (France), <Jean-Eudes.Arlot@obspm.fr>

URL: <http://www.imcce.fr/host/iau_wgnps/iauwg.html> 


\section{DIVISION IV - Stars}

URL: <http://www.galax.obspm.fr/IAUdiv4>

\section{President}

Monique Spite

GEPI

Observatoire de Paris-Meudon

5 Place J. Janssen

F-92195 Meudon Cedex

France

Phone: +33145077839

Fax: +33145077878

Email: <monique.spite@obspm.fr>

Email: <ccorbally@as.arizona.edu>
Vice-President

Christopher J. Corbally

Vatican Observatory Research Group

Steward Observatory

University of Arizona

933 North Cherry Avenue

Tucson, AZ 85721

USA

Phone: +1520621 3225

Fax: +1 5206211532

\section{Organizing Committee}

Christine Allen, P-C26 (Mexico), Francesca d'Antona, P-C35 (Italy), Dainis Dravins, PP (Sweden), Sunetra Giridhar, P-C45 (India), John D. Landstreet, P-C36 (Canada), and Mudumba Parthasarathy, P-C29 (India)

\section{PARTICIPATING COMMISSIONS AND COMMISSION WORKING GROUPS}

\section{Div.IV/Comm.26 - Double and Multiple Stars}

P: Christine Allen (Mexico), <chris@astroscu.unam.mx>

VP: Jose A.D. Docobo (Spain), <oadoco@usc.es>

OC: Yuri Y. Balega (Russia) John Davis (Australia), William I. Hartkopf, PP (USA), Brian D. Mason (USA), Edouard Oblak (France), Terry D. Oswalt (USA), Dimitri Pourbaix (Belgium), and Colin D. Scarfe (Canada)

URL: <http://ad.usno.navy.mil/wds/dsl.html>

\section{Div.IV/Comm.26/WG - Binary and Multiple System Nomenclature}

Chair: Brian D. Mason (USA), <wih@usno.navy.mil>

URL: <http://ad.usno.navy.mil/wds/newwds.html>

\section{Div.IV/Comm.29 - Stellar Spectra}

P: Mudumba Parthasarathy (India), <partha@iiap.res.in>

VP: Nikolai E. Piskunov (Sweden), < piskunov@astro.uu.se>

OC: Fiorella Castelli (Italy), Kenneth G. Carpenter (USA), Katia Cunha (Brazil), Philippe R.J. Eenens (Mexico), Ivan Hubeny (USA), Sylvia C.F. Rossi (Brazil), Chris Sneden, PP (USA), Masahide Takada-Hidai (Japan), Glenn M. Wahlgren (Sweden), and Werner W. Weiss (Austria)

URL: <http://www.iiap.res.in/personnel/partha/IAUcom29.html>

\section{Div.IV/Comm.35 - Stellar Constitution}

P: Francesca d'Antona (Italy), <dantona@mporzio.astro.it>

VP: Corinne Charbonnel (Switzerland), <Corinne.Charbonnel@obs.unige.ch>

OC: Gilles Fontaine (Canada), Richard B. Larson (USA), John Lattanzio (Australia), James W. Liebert (USA), Ewald Mueller (Germany), Achim Weiss (Germany), and Lev R. Yungelson (Russia)

URL: <http://iau-c35.stsci.edu> 


\section{Div.IV/Comm.36 - Theory of Stellar Atmospheres}

P : John D. Landstreet (Canada), <jlandstr@uwo.ca>

VP: Martin Asplund (Australia), <martin@mso.anu.edu.au>

OC: Suchitra C. Balachandran (USA), Svetlana V. Berdyugina (Switzerland), Peter H. Hauschildt (Germany), Hans G. Ludwig (France), Lyudmila I. Mashonkina (Russia), K.N. Nagendra (India), Joachim Puls (Germany), Sofia Randich (Italia), Monique Spite, PP (France), and Grazina Tautvaisiene (Lithuania)

URL: <http://www.galax.obspm.fr/IAU36/>

\section{Div.IV/Comm.45 - Stellar Classification}

P: Sunetra Giridhar (India), < giridhar@iiap.res.in>

VP: Richard O. Gray (USA), <grayro@appstate.edu>

OC: Coryn A.L. Bailer-Jones (Germany), Christopher J. Corbally, PP (USA), Laurent Eyer (Switserland), Michael J. Irwin (UK), Joseph D. Kirkpatrick (USA), Steven Majewski (USA), Dante Minniti (Chile), and Birgitta Nordström (Denmark)

URL: <http://www.iap.fr/SitesHeberges/com45uai/index.html>

\section{DIVISION IV WORKING GROUPS}

\section{Div.IV/WG - Massive Stars}

Chair: Stanley P. Owocki (USA), <owocki@bartol.udel.edu>

URL: <http://www.astroscu.unam.mx/massive_stars/index.php>

\section{Div.IV/WG - Abundances in Red Giants}

Chair: John Lattanzio (Australia), <john.lattanzio@sci.monash.edu.au>

URL: <http://www.maths.monash.edu.au/ johnl/wgarg/>

\section{DIVISION IV INTER-DIVISION WORKING GROUPS}

\section{Div.IV-V/WG - Active OB Stars}

co-Chair: Juan Fabregat (Spain), <Juan.Fabregat@uv.es>

co-Chair: Geraldine J. Peters (USA), < gjpeters@mucen.usc.edu>

URL: <http://www.astro.virginia.edu/ dam3ma/benews/iauwg_abs.html>

\section{Div.IV-V/WG - Ap and Related Stars}

Chair: Margarida Cunha (Portugal), <mcunha@astro.up.pt>

URL: <http://www.eso.org/gen-fac/pubs/apn/apwg/>

\section{Div.IV-V-IX/WG - Standard Stars}

Chair: Christopher J. Corbally (USA), <ccorbally@as.arizona.edu>

URL: <http://stellar.phys.appstate.edu/ssn/> 
DIVISION V - Variable Stars

URL: <http://www.konkoly.hu/IAUDV/>

President

Alvaro Giménez

Centro de Astrobiologia

INTA / CSIC

Carretera de Torrejon a Ajalvir

Torreon de Ardoz

E-28850 Madrid

Spain

Phone/Fax: +34 915201111

Email: <agimenez@rssd.esa.int>

\section{Vice-President}

Steven D. Kawaler

Department of Physics and Astronomy

Iowa State University

A323 Zaffarano Hall

Ames, IA 50011-3160

USA

Phone: +1 5152949728

Fax: +1 5152946027

Email: <sdk@iastate.edu>

\section{Secretary}

Conny Aerts (Belgium), <conny@ster.kuleuven.ac.be>

\section{Organizing Committee}

Jørgen Christensen-Dalsgaard,PP (Denmark), Edward F. Guinan (USA), Donald W. Kurtz (UK), and Slavek M. Rucinski, P-C42 (Canada)

\section{PARTICIPATING COMMISSIONS}

\section{Div.V/Comm.27 - Variable Stars}

P: Steven D. Kawaler (USA), <sdk@iastate.edu >

VP: Gerald Handler (Austria), <handler@astro.univie.ac.at>

OC: Conny Aerts (Belgium), Timothy R. Bedding (Australia), Márcio Catelán (Chile), Margarida Cunha (Portugal), Laurent Eyer (Switzerland), C. Simon Jeffery (N. Ireland), Peter Martinez (South Africa), Katalin Olah (Hungary), Karen Pollard (New Zealand), and Seetha Somasundaram (India)

URL: <http://www.konkoly.hu/IAUC27>

\section{Div.V/Comm.42 - Close Binary Stars}

P: Slavek Rucinski (Canada), <rucinski@astro.utoronto.ca>

VP: Ignasi Ribas (Spain), <iribas@ieec.uab.es>

OC: Alvaro Giménez, PP (Spain), Petr Harmanec (Czech Rep.), Ronald W. Hilditch (UK), Janusz Kaluzny (Poland), Panayiotis Niarchos (Greece), Birgitta Nordström (Denmark), Katalin Olah (Hungary), Mercedes T. Richards (USA), Colin D. Scarfe, PP (Canada), Edward M. Sion (USA), Guillermo Torres (USA), and Sonja Vrielmann (Germany)

URL: <http://www.konkoly.hu/IAUDV/>

\section{DIVISION V WORKING GROUP}

\section{Div.V/WG - Spectroscopic Data Archiving}

Chair: Elizabeth Griffin (Canada), <Elizabeth.Griffin@nrc-cnrc.gc.ca>

URL: <http://www.konkoly.hu/SVO/>

\section{INTER-DIVISION WORKING GROUPS}

\section{Div.IV-V/WG - Active OB Stars}

co-Chair: Juan Fabregat (Spain), <Juan.Fabregat@uv.es> 
co-Chair: Geraldine J. Peters (USA), < gjpeters@mucen.usc.edu>

URL: <http://www.astro.virginia.edu/ dam3ma/benews/iauwg_abs.html>

Div.IV-V/WG - Ap and Related Stars

Chair: Margarida Cunha (Portugal) <mcunha@astro.up.pt $>$

URL: <http://www.eso.org/gen-fac/pubs/apn/apwg/>

\section{Div.IV-V-IX/WG - Standard Stars}

Chair: Christopher J. Corbally (USA), <ccorbally@as.arizona.edu>

URL: <http://stellar.phys.appstate.edu/ssn/> 


\section{DIVISION VI - Interstellar Matter}

URL: <www.div6.qub.ac.uk>

\section{President}

School of Mathematics and Physics

Faculty of Engineering \& Physical Sciences

Queen's University Belfast

13 Stranmillis Road

Belfast BT9 5AF

Northern Ireland

Phone: +44 2890976523

Fax: +44 2890974536

E-mail: <Tom.Millar@qub.ac.uk>

\section{Vice-President}

Astronomy Department

University of Illinois

103 Astronomy Building

1002 West Green Street

Urbana, IL 61801

USA

Phone: +1 2173335535

Fax: +1 2172447638

Email: <chu@astro.uiuc.edu>

\section{Organizing Committee}

Dieter Breitschwerdt (Germany), Michael G. Burton (Australia), Sylvie Cabrit (France), Paola Caselli (Italia), John E. Dyson, PP (UK), Gary J. Ferland (USA), Elisabete M.de Gouveia Dal Pino (Brazil), Mika J. Juvela (Finland), Bon-Chul Koo (S. Korea), Sun Kwok (Hong Kong), Susana Lizano (Mexico), Michael Rozyczka (Poland), L. Viktor Toth (Hungary), Masato Tsuboi (Japan), and Ji Yang (China Nanjing)

\section{PARTICIPATING COMMISSION}

Div.VI/Comm.34 - Interstellar Matter

$\mathrm{P}=\mathrm{P}$ Division VI, VP $=$ VP Division VI, OC $=$ OC Division VI

\section{DIVISION VI WORKING GROUPS}

\section{Div.VI/WG - Star Formation}

Chair: Franceso Palla (Italy), <palla@arcetri.astro.it>

URL: <http://www.arcetri.astro.it/sfwg/>

Div.VI/WG - Astrochemistry

Chair: Ewine F. van Dishoeck (Netherlands), < ewine@strw.leidenuniv.nl>

URL: <http://www.strw.leidenuniv.nl/iau34/>

Div.VI/WG - Planetary Nebulae Chair: Arturo Manchado (Spain), <amt@iac.es> URL: <http://www.iac.es/proyect/PNgroup/wg/> 


\section{DIVISION VII - Galactic System}

URL: <http://www.ari.uni-heidelberg.de/interessantes/iaudivisionVII/IAUDivVII.html>

\section{President}

Ortwin Gerhard

MPI für Extraterrestrische Physik

Giessenbachstrasse 1

D-85748 Garching bei München

Germany

Phone: +49 $89300003539 / 3503$

Fax: +49 89300003351

Email: <gerhard@mpe.mpg.de>

\section{Vice-President}

Despina Hatzidimitriou

Department of Physics

University of Crete

P.O. Box 2208

GR-710 03 Heraklion

Greece

Phone: +30 2810394212

Fax: +30 2810394201

Email: <dh@physics.uoc.gr>

\section{Organizing Committee}

Charles J. Lada, VP-C37 (USA), Ata Sarajedini (USA), Patricia A. Whitelock, PP (South Africa), Rosemary F. Wyse, VP-C33 (USA), and Joseph Lazio (USA)

\section{PARTICIPATING COMMISSIONS}

\section{Div.VII/Comm.33 - Structure and Dynamics of the Galactic System}

P: Ortwin Gerhard (Germany), < gerhard@mpe.mpg.de>

VP: Rosemary F. Wyse (USA), <wyse@pha.jhu.edu>

OC: Yuri N. Efremov (Russia), Wyn Evans (UK), Chris Flynn (Finland), Jonathan E. Grindlay (USA), Joseph Lazio (USA), Birgitta Nordström (Denmark), Patricia A. Whitelock (South Africa), and Chi Yuan (South Korea)

URL: <http://www.saao.ac.za/IAU/IAUComm33.html>

\section{Div.VII/Comm. 37 Star - Clusters and Associations}

P: Despina Hatzidimitriou (Greece), <dh@physics.uoc.gr $>$

VP: Charles J. Lada (USA), <clada@cfa.harvard.edu >

OC: Russell D. Cannon (Australia), Kyle McC. Cudworth (USA), Gary S. Da Costa (Australia), LiCai Deng (China), Young-Wook Lee (South Korea), Ata Sarajedini (USA), and Monica Tosi (Italy)

URL: <http://www.ari.uni-heidelberg.de/interessantes/iaucommission37/

IAUComm37.html $>$

\section{DIVISION VII WORKING GROUP}

\section{Div.VII/WG - Galactic Center}

Chair: Joseph Lazio (USA), <Lazio@nrl.navy.mil>

URL: <http://www.aoc.nrao.edu/gcnews/> 


\section{DIVISION VIII - Galaxies and the Universe}

URL: <http://www.star.bris.ac.uk/iau/>

\section{President}

Sadanori Okamura

Department of Astronomy

School of Science

University of Tokyo

7-3-1 Hongo Bunkyo-ku

Tokyo 113-0033

Japan

Phone: +8135841 4257

Fax: +81358417644

Email: <okamura@astron.s.u-tokyo.ac.jp>

\section{Organizing Committee}

Francesco Bertola, PP (Italy), Françoise Combes, P-C28 (France), Roger L. Davies, VPC28 (UK), Thanu Padmanabhan, VP-C47 (India), and Rachel L. Webster, P-C47 (Australia)

\section{Webmaster}

Mark Birkinshaw (UK), <Mark.Birkinshaw@bristol.ac.uk>

\section{PARTICIPATING COMMISSIONS}

\section{Div.VIII/Comm.28 - Galaxies}

P: Franoise Combes (France), <francoise.combes(@)obspm.fr >

VP: Roger L. Davies (UK), <roger.davies@durham.ac.uk>

OC: Avishai Dekel (Israel), Marijn Franx (Netherlands), John S. Gallagher (USA), Naomasa Nakai (Japan), Monica Rubio (Chile), Valentina Karachentseva (Ukraine), Gillian R. Knapp (USA), Renée C. Kraan-Korteweg (South Africa), Bruno Leibundgut (Germany), Jayant V. Narlikar (India), and Elaine M. Sadler (Australia)

URL: < http://aramis.obspm.fr/IAU28/index.php>

\section{Div.VIII/Comm.47 - Cosmology}

P: Rachel L. Webster (Australia), <rwebster@physics.unimelb.edu.au>

VP: Thanu Padmanabhan (India), <paddy@iucaa.ernet.in>

OC: $($ tbd)

URL: $<\operatorname{tbd}>$

\section{DIVISION VIII WORKING GROUP}

\section{Div.VIII/WG - Supernovae}

co-Chair: Brian Schmidt (Australia), <brian@mso.anu.edu.au>

co-Chair: Wolfgang Hillebrandt (Germany), <wfh@MPA-Garching.MPG.de>

URL: <http://www.star.bris.ac.uk/iau/news/sn_wg_ga_26.pdf> 


\section{DIVISION IX - Optical and Infrared Techniques}

URL: <http://www.ifa.hawaii.edu/users/kud/iau/DivIX.htm>

\section{President}

Rolf-Peter Kudritzki

Institute for Astronomy

2680 Woodlawn Drive

Honolulu, HI 96822

USA

Phone: +18089568566

Fax: +1 8089463467

Email: <kud@ifa.hawaii.edu>

\author{
Vice-President \\ Andreas Quirrenbach \\ Landessternwarte, Univerity of Heidelberg \\ Koenigstuhl 12 \\ D-69117 Heidelberg \\ Germany \\ Phone: +49 6221541792 \\ Fax: +496221 541702 \\ Email: <A.Quirrenbach@lsw.uni-heidelberg.de>
}

\section{Organizing Committee}

Michael G. Burton WG A (Australia), Xiangqun Cui (China), Martin Cullum (Germany), Peter Martinez P-C25 (South Africa), Guy S. Perrin P-C54 (France), Andrei A. Tokovinin (Chile), Guillermo Torres VP-C30 (USA), and Stephane Udry P-C30 (Switzerland)

\section{Consultants}

Christiaan L. Sterken (Belgium) and Michel Dennefeld (France)

\section{PARTICIPATING COMMISSIONS AND COMMISSION WORKING GROUPS}

\section{Div.IX/Comm.25 - Stellar Photometry and Polarimetry}

P: Peter Martinez (South Africa), <peter@saao.ac.za>.

VP: Eugene F. Milone (Canada), <milone@ucalgary.ca $>$.

OC: Arlo U. Landolt, PP (USA), Edward G. Schmidt (USA), Carme Jordi (Spain), Christiaan L. Sterken (Belgium), Alexei Mironov (Russia), and Qian Sheng-Bang (China) URL: <http://www.vub.ac.be/STER/IAU/IAUComm25.html>

\section{Div.IX/Comm.25 - WG Infrared Astronomy}

Chair: Eugene F. Milone (Canada), <milone@ucalgary.ca $>$

URL: <http://www.ucalgary.ca/ milone/IRWG/>

\section{Div.IX/Comm.30 - Radial Velocities}

P: Stephane Udry (Switzerland), <stephane.udry@obs.unige.ch>

VP: Guillermo Torres (USA), <torres@cfa.harvard.edu>

OC: Francis C. Fekel (USA), Kenneth C. Freeman (Australia), Elena V. Glushkova (Russia), Goeffrey W. Marcy (USA), Robert D. Mathieu (USA), Birgitta Nordström, PP (Denmark), Dimitri Pourbaix (Belgium), Catherine Turon (France), and Tomaz Zwitter (Slovenia)

URL: <http://www.ctio.noao.edu/science/iauc30/iauc30.html>

\section{Div.IX/Comm.30 WG - Radial-Velocity Standard Stars}

Chair: Stephane Udry (Switzerland), <stephane.udry@obs.unige.ch>

URL: <http://obswww.unige.ch/ udry/std/std.html>

Div.IX/Comm.30 WG - Stellar Radial Velocity Bibliography

Chair: Hugo Levato (Argentina), <hlevato@casleo.gov.ar>

URL: <www.casleo.gov.ar/indexingles.htm> 
Div.IX/Comm.30 WG - Catolog of Orbital Elements of Spectroscopic Binary Systems

Chair: Dimitri Pourbaix (Belgium), <pourbaix@astro.ulb.ac.be>

URL: <http://sb9.astro.ulb.ac.be/>

Div.IX/Comm.54 - Optical and Infrared Interferometry

P: Guy S. Perrin (France), <guy.perrin@obspm.fr>

VP: Stephen T. Ridgway (USA), <ridgway@noao.edu>

S : Gerard T. van Belle (USA), < gerard(@)ipac.caltech.edu>

OC: Gilles Duvert (France), Reinhard Genzel (Germany), Christopher Haniff (UK), Christian A. Hummel (Germany), Peter R. Lawson (USA), John D. Monnier (USA), Didier Queloz (Switzerland), Peter G. Tuthill (Australia), and Farrokh Vakili (France) URL: <http://olbin.jpl.nasa.gov/iau/2006/commission.html>

DIVISION IX WORKING GROUPS

Div.IX/WG - Adaptive Optics

Chair: (tbd)

Div.IX/WG - Site Testing Instruments

Chair: Andrei A. Tokovinin (Chile), <atokovinin@ctio.noao.edu>

\section{Div.IX/WG - Large Telescope Projects}

Chair: (tbd)

Div.IX/WG - Small Telescope Projects

Chair: (tbd)>

Div.IX/WG - Detectors

Chair: Tim Abbott (USA), <tabbott@ctio.noao.edu>

URL: <http://www.ctio.noao.edu/CCD-world/>

Div.IX/WG - Sky Surveys

Chair: Quentin Parker (Australia), <qap@ics.mq.edu.au>

URL: <http://alba.stsci.edu/wgss/>

INTER-DIVISION WORKING GROUPS

Div.IV-V-IX/WG - Standard Stars

Chair: Christopher J. Corbally (USA), <ccorbally@as.arizona.edu>

URL: <http://stellar.phys.appstate.edu/ssn/>

Div.IX-X/WG Encouraging the International Development of Antarctic Astronomy

Chair: Michael G. Burton (Australia), <mgb@phys.unsw.edu.au>

URL: <www.phys.unsw.edu.au/jacara/iau>

Div.IX-X-XI/WG - Astronomy from the Moon

Chair: Sallie L. Baliunas (USA), <baliunas@cfa.harvard.edu> vice-Chair: Yoji Kondo (USA), <kondo@stars.gsfc.nasa.gov>

URL: <http://www.cfa.harvard.edu/moon/> 
DIVISION X - Radio Astronomy

URL: <http://www.bao.ac.cn/IAU_COM40/>

President

Rendong Nan

National Astronomical Observatories

Chinese Academy of Sciences

A20 Datun Road, Chaoyang District

Beijing 100012

P.R. China

Phone: +86106487 7280

Fax: +86106485 2055

Email: <nrd@bao.ac.cn>
Vice-President

Russell A. Taylor

Department of Physics and Astronomy

University of Calgary

2500 University Drive NW

Calgary AB T2N $1 \mathrm{~N} 4$

Canada

Phone: +1 4032205385

Fax: +1 4032893331

Email: <russ@ras.ucalgary.ca>

\section{Organizing Committee}

Christopher L. Carilli (USA), Jessica Chapman (Australia), Gloria M. Dubner (Argentina), Michael Garrett (Netherlands), W. Miller Goss (USA), Richard E. Hills (UK), Hisashi Hirabayashi (Japan), Luis F. Rodriguez, PP (Mexico), Prajval Shastri (India), and Jose M. Torrelles (Spain)

\section{PARTICIPATING COMMISSION}

Div.X/Comm.40 - Radio Astronomy

$\mathrm{P}=\mathrm{P}$ Division $\mathrm{X}, \mathrm{VP}=\mathrm{VP}$ Division $\mathrm{X}, \mathrm{OC}=\mathrm{OC}$ Division $\mathrm{X}$

\section{DIVISION X WORKING GROUPS}

\section{Div.X/WG - Global VLBI}

Chair: Jonathan D. Romney (USA), <jromney@nrao.edu>

URL: <http://www.bao.ac.cn/IAU_COM40/WG/WgVLBI.html>

\section{Div.X/WG - Interference Mitigation}

Chair: Anastasios Tzioumis (Australia), <atzioumi@atnf.csiro.au>

URL: <http://www.bao.ac.cn/IAU_COM40/WG/WgRF.html>

\section{Div.X/WG - Astrophysically Important Spectral Lines}

Chair: Masatoshi Ohishi (Japan), <ohishi@nao.ac.jp>

URL: <http://www.bao.ac.cn/IAU_COM40/WG/WgSL.html>

\section{DIVISION X INTER-DIVISION WORKING GROUPS}

\section{Div.IX-X/WG - Encouraging the International Development of Antarctic} Astronomy

Chair: Michael G. Burton (Australia), <mgb@phys.unsw.edu.au>

URL: <http://www.bao.ac.cn/IAU_COM40/WG/WgAntarc.html>

\section{Div.IX-X-XI WG - Astronomy from the Moon}

Chair: Sallie Baliunas (USA), <baliunas@cfa.harvard.edu>

vice-Chair: Yoji Kondo (USA), < kondo@stars.gsfc.nasa.gov>

URL: <http://www.cfa.harvard.edu/moon/>

\section{Div.X-XII/WG - Historic Radio Astronomy}

Chair: Wayne Orchiston (Australia), <Wayne.Orchiston@jcu.edu.au>

URL: <http://www.bao.ac.cn/IAU_COM40/WG/WgHistRA.html> 


\section{DIVISION XI - Space and High Energy Astrophysics}

URL: < http://www.mpe.mpg.de/IAU_DivXI/

\section{President}

Günther Hasinger

MPI für Extraterrestrische Physik

Giessenbachstrasse 1

D-85748 Garching-bei-München

Germany

Phone: +49 89300003401

Fax: +49 89300003404

Email: <ghasinger@mpe.mpg.de>
Vice-President

Christine Jones

Harvard-Smithonian Center for Astrophysics

60 Garden Street

Cambridge, MA 02138

USA

Phone: +1 6174957137

Fax: +1 6174957356

Email: <cjf@cfa.harvard.edu>

\section{Organizing Committee}

João Braga (Brazil), Noah Brosch (Israel), Thijs de Graauw (Chile), Leonid I. Gurvits (Netherlands), George Helou (USA), Ian D. Howarth (UK), Hideyo Kunieda (Japan), Thierry Montmerle (France), Haruyuki Okuda (Japan), Marco Salvati (Italy), and Kulinder Pal Singh (India)

\section{PARTICIPATING COMMISSION}

Div.XI/Comm.44 - Space and High Energy Astrophysics

$\mathrm{P}=\mathrm{P}$ Division XI, VP = VP Division XI, OC = OC Division XI

\section{DIVISION XI WORKING GROUP}

Div.XI/WG - Particle Astrophysics

Chair: Reinhard Schlickeiser (Germany), <office@tp4.ruhr-uni-bochum.de>

URL: < http://iau.physik.rub.de/ >

\section{DIVISION XI INTER-DIVISION WORKING GROUP}

\section{Div.IX-X-XI/WG - Astronomy from the Moon}

Chair: Sallie Baliunas (USA), < baliunas@cfa.harvard.edu >.

Vice-Chair: Yoji Kondo (USA), < kondo@stars.gsfc.nasa.gov >

OC: Oddbjorn Engvold (Norway), Karel A. van der Hucht (Netherlands), Norio Kaifu (Japan), Haruyuki Okuda (Japan), and Yervant Terzian (USA)

URL: < http://www.cfa.harvard.edu/moon/ > 
DIVISION XII - Union-Wide Activities

URL: <http://www.iaudivisionxii.org/>

President

Malcolm G. Smith

AURA / CTIO / NOAO

Casilla 603

La Serena

Chile

Phone: +56 51205217

Fax: +56 51205356

Email: <msmith@ctio.noao.edu>
Vice-President

Françoise Genova

Observatoire Astronomique

Université de Strasbourg

11 rue de l'Université

F-67000 Strasbourg

France

Phone: +33 390242476

Fax: +33 390242432

Email: <genova@astro.u-strasbg.fr>

\section{Secretary / Webmaster}

Lars Lindberg Christensen (ESO/ESA), <lars@eso.org>

\section{Organizing Committee}

Johannes Andersen, PP (Denmark), Steven R. Federman, P-C14 (USA), Alan C. Gilmore, P-C6 (New Zealand), Raymond P. Norris, P-C5 (Australia), Ian E. Robson, P-C55 (UK), Il-Seong Nha, P-C41 (South Korea), Magda G. Stavinschi, P-C46 (Romania), Richard J. Wainscoat, VP-C50 (USA), and Virginia L. Trimble, PP (USA)

\section{PARTICIPATING COMMISSIONS AND COMMISSION WORKING GROUPS}

\section{Div.XII/Comm.5 - Documentation and Astronomical Data}

P : Raymond P. Norris, WG AD (Australia), <Ray.Norris@csiro.au>

VP: Masatoshi Ohishi (Japan), <ohishi@nao.ac.jp>

OC: Françoise Genova, PP (France), Uta Grothkopf, WG Libraries (Germany), Oleg Yu. Malkov (Russia), Masatoshi Ohishi (Japan), William D. Pence, WG FITS (USA), Marion Schmitz, WG Nomen. (USA), Robert J. Hanisch, WG VO (USA), and Xu Zhou (China)

URL: < http://www.atnf.csiro.au/people/rnorris/IAUC5/>

\section{Div.XII/Comm.5/WG - Astronomical Data}

Chair: Raymond P. Norris (Australia), <Ray.Norris@csiro.au>

URL: < http://www.atnf.csiro.au/people/rnorris/WGAD/>

\section{Div.XII/Comm.5/WG - Nomenclature}

Chair: Marion Schmitz (USA), <zb4ms@ipac.caltech.edu>

URL: <http://vizier.u-strasbg.fr/Dic/iau-spec.htx>

\section{Div.XII/Comm.5/WG - Libraries}

Chair: Uta Grothkopf (Germany), <ugrothko@eso.org>

URL: <http://www.eso.org/gen-fac/libraries/IAU-WGLib/>

\section{Div.XII/Comm.5/WG - FITS Data Format}

Chair: William D. Pence (USA), <William.D.Pence@nasa.gov> vice-Chair: François Ochsenbein (France), < francois@astro.u-strasbg.fr > URL: < http://fits.gsfc.nasa.gov/iaufwg/ >

Div.XII/Comm.5/WG - Virtual Observatories, Data Centers and Networks Chair: Robert J. Hanisch (USA), < hanisch@stsci.edu $>$ URL: < http://cdsweb.u-strasbg.fr/IAU/wgvo.html > 
Div.XII/Comm.5/TF - Preservation and Digitization of Photographic Plates Chair: Elizabeth Griffin (Canada), <Elizabeth.Griffin@hia-iha.nrc-cnrc.gc.ca> URL: < http://www.lizardhollow.net/PDPP.htm >

\section{Div.XII/Comm.6 - Astronomical Telegrams}

P: Alan C. Gilmore (New Zealand), < alan.gilmore@canterbury.ac.nz>

VP: Nicolay Samus (Russia), <samus@lnfm1.sai.msu.ru>

OC: Brian G. Marsden (USA), Daniel W.E. Green (USA), Syuichi Nakano (Japan), Elizabeth Roemer (USA), Jana Ticha (Czech Rep.), Hitoshi Yamaoka (Japan), and Kaare Aksnes (Norway)

URL: <http://cfa-www.harvard.edu/iau/Commission6.html>

\section{Div.XII/Comm.6/Service - Central Bureau for Astronomical Telegrams}

Chair: Daniel W. E. Green (USA), <dgreen@cfa.harvard.edu>

URL: <http://cfa-www.harvard.edu/iau/cbat.html>

Div.XII/Comm.14 - Atomic and Molecular Data

P: Steven R. Federman (USA), <steven.federman@utoledo.edu>

VP: Glenn M. Wahlgren (Sweden), <glenn.wahlgren@astro.lu.se>

OC: Sveneric Johansson (Sweden), Milan Dimitrijevic (Serbia), Alain Jorissen (Belgium), Lyudmila I. Mashonkina (Russia), Farid Salama (USA), Jonathan Tennyson (UK), and Ewine F. van Dishoeck (Netherlands)

URL: <http://www.astro.lu.se/Research/astrophys/iau/>

Div.XII/Comm.14/WG - Atomic Data

co-Chair: Gillian Nave, (USA), <gnave@nist.gov>

co-Chair: Glenn M. Wahlgren (Sweden), < glenn.wahlgren@astro.lu.se>

co-Chair: Jeffrey R. Fuhr (USA), < jeffrey.fuhr@nist.gov>

URL: <http://iacs.cua.edu/IAUC14>

\section{Div.XII/Comm.14/WG - Molecular Data}

Chair: John H. Black (Sweden), <John.Black@chalmers.se>

URL: <http://iacs.cua.edu/IAUC14>

\section{Div.XII/Comm.14/WG - Collision Processes}

Chair: Philip Stancil (USA), <stancil@physast.uga.edu>

co-Chair: Gillian Peach (UK), < ucap22g@ucl.ac.uk>

URL: <http://iacs.cua.edu/IAUC14/>

Div.XII/Comm.14/WG - Solids and their Surfaces

Chair: G. Vidali

URL: <http://iacs.cua.edu/IAUC14/>

\section{Div.XII/Comm.41 - History of Astronomy}

P: Il-Seong Nha (South Korea), <slisnha@chol.com>

VP: Clive L.N. Ruggles (UK), <rug@le.ac.uk>

OC: Alexander A. Gurshtein (Russia/USA), Teije de Jong (Netherlands), Rajesh K. Kochhar (India), Tsuko Nakamura (Japan), Wayne Orchiston (Australia), Antonio A.P. Videira (Brazil), and Brian Warner (South Africa)

URL: <http://www.le.ac.uk/has/c41/>

\section{Div.XII/Comm.41/WG - Archives}

Chair: Ileana Chinnici (Italy), <chinnici@astropa.unipa.it>

URL: <http://www.le.ac.uk/has/c41/wgarc.html> 
Div.XII/Comm.41/WG - Historical Instruments

Chair: Luisa Pigatto (Italy), <luisa.pigatto@oapd.inaf.it>

URL: <http://www.oapd.inaf.it/museo/PagineInglesi/History

Div.XII/Comm.41/WG - Transits of Venus

Chair: Steven J. Dick (USA), <steven.j.dick@nasa.gov>

URL: <http://www.le.ac.uk/has/c41/wgtov.html>

Div.XII/Comm.46 - Astronomy Education and Development

P: Magdalena G. Stavinschi (Romania), <magda@aira.astro.ro>

VP: Rosa M. Ros (Spain), <ros@mat.upc.es>

OC: Michèle Gerbaldi (France), Edward F. Guinan (USA), John B. Hearnshaw (New Zealand), Margarita Metaxa (Greece), Nidia Morrell (Argentina), Mazlan Othman (Malaysia), John R. Percy (Canada), Charles R. Tolbert (USA), Silvia Torres-Peimbert (Mexico), and James C. White (USA)

URL: <http://physics.open.ac.uk/IAU46/>

Div.XII/Comm.46/PG - World Wide Development of Astronomy

Chair: John B. Hearnshaw (New Zealand), <john.hearnshaw@canterbury.ac.nz>

URL: <http://physics.open.ac.uk/IAU46/programme\%20groups.html\#PGWWDA>

Div.XII/Comm.46/PG - Teaching for Astronomy Development

co-Chair: Laurence A. Marshall (USA), <marshall@gettysburg.edu>

co-Chair: Edward F. Guinan (USA), <edward.guinan@villanova.edu>

URL: <http://physics.open.ac.uk/IAU46/programme\%20groups.html\#TAD>

$<$ http://physics.open.ac.uk/IAU46/guidelines.html>

Div.XII/Comm.46/PG - International Schools for Young Astronomers

Chair: Jean-Pierre de Greve (Belgium), <jpdgreve@vub.ac.be>

vice-Chair: Kam-Ching Leung (USA), <kleung@unlserve.unl.edu>

URL: <http://physics.open.ac.uk/IAU46/programme\%20groups.html\#ISYA>

Div.XII/Comm.46/PG - Collaborative Programs

Chair: Hans J. Haubold (Austria), <haubold@kph.tuwien.ac.at>

URL: <http://physics.open.ac.uk/IAU46/programme\%20groups.html\#CP>

Div.XII/Comm.46/PG - Exchange of Astronomers

Chair: John R. Percy (Canada), <jpercy@credit.erin.utoronto.ca>

URL: <http://physics.open.ac.uk/IAU46/programme\%20groups.html\#EoA>

Div.XII/Comm.46/PG - National Liaison on Astronomy Education

Chair: Barrie W. Jones (UK), <b.w.jones@open.ac.uk>

URL: <http://physics.open.ac.uk/IAU46/programme\%20groups.html\#NL>

Div.XII/Comm.46/PG - Commission Newsletter

Chair: Barrie W. Jones (UK), <b.w.jones@open.ac.uk>

URL: <http://physics.open.ac.uk/IAU46/programme\%20groups.html\#News>

Div.XII/Comm.46/PG - Public Education on the Occasions of Solar Eclipses

Chair: Jay M. Pasachoff (USA), <jay.m.pasachoff@williams.edu>

URL: <http://physics.open.ac.uk/IAU46/programme\%20groups.html\#PGSE>

Div.XII/Comm.46/PG - Exchange of Books and Journals

Chair: Susana E. Deustua (USA), <deustua@aas.org >

URL: <http://physics.open.ac.uk/IAU46/programme\%20groups.html\#Ex> 
Div.XII/Comm.50 - Protection of Existing and Potential Observatory Sites P : Hugo E. Schwarz (Chile, $†$ 2006)

VP: Richard J. Wainscoat, (USA), acting president ,<rjw@ifa.hawaii.edu $>$

OC: Carlo Blanco (Italy), Jim Cohen, PP (UK, † 2006), David L. Crawford (USA), Magarita Metaxa (Greece), and Woodruff T. Sullivan, PP (USA)

URL: <http://www.ctio.noao.edu/cgi-bin/iau50.pl>

\section{Div.XII/Comm.50/WG - Controlling Light Pollution}

Chair: Richard J. Wainscoat (USA), <rjw@ifa.hawaii.edu>

URL: <http://www.ctio.noao.edu/light_pollution/iau50/>

Div.XII/Comm.55 - Communicating Astronomy with the Public

P : Ian E. Robson (UK), <eir@roe.ac.uk>

VP: Dennis Crabtree (Canada), <Dennis.Crabtree@nrc-cnrc.gc.ca $>$

S : Lars Lindberg Christensen (ESO/ESA), <lars@eso.org>

OC: Augusto Damineli Neto (Brazil), Richard T. Fienberg (USA), Anne Green (Australia), Ajit K. Kembhavi (India), Birgitta Nordström (Denmark), Oscar Alvarez-Pomares (Cuba), Kazuhiro Sekiguchi (Japan), Patricia A. Whitelock (South Africa), and Jin Zhu (China)

URL: <http://www.communicatingastronomy.org/>

Div.XII/Comm.55/WG - Washington Charter

Chair: Dennis Crabtree (Canada), <Dennis.Crabtree@nrc.ca>

URL: <http://www.communicatingastronomy.org/washington_charter/index.html>

Div.XII/Comm.55/WG - Virtual Astronomy Multimedia Project

Chair: Adrienne Gaulthier (USA), <gauthier@as.arizona.edu>

URL: <http://www.communicatingastronomy.org/repository/index.html>

Div.XII/Comm.55/WG - Best practices

Chair: Lars Lindberg Christensen (Germany), <lars@eso.org >

URL: <http://www.communicatingastronomy.org/bestpractices/index.html>

Div.XII/Comm.55/WG - Communicating Astronomy Journal

Chair: Pedro Russo (Germany), <prusso@eso.org >

URL: <http://www.communicatingastronomy.org/journal/index.html>

Div.XII/Comm.55/WG - New Ways of Communicating Astronomy with the Public

Chair: Michael West (USA), <mwest@gemini.edu>

URL: <http://www.communicatingastronomy.org/newways/index.html>

Div.XII/Comm.55/WG - Communicating Astronomy with the Public conferences

Chair: Ian E. Robson (UK), <eir@roe.ac.uk>

URL: <http://www.communicatingastronomy.org/capconferences/index.html>

\section{DIVISION XII INTER-DIVISION WORKING GROUP}

\section{Div.X-XII/WG - Historic Radio Astronomy}

Chair: Wayne Orchiston (Australia), <Wayne.Orchiston@jcu.edu.au>

URL: <http://www.le.ac.uk/has/c41/wghra.html> 